\title{
External Silane Donors in Ziegler-Natta Catalysis. A Three-Site Model Analysis of Effects on Catalyst Active Sites
}

\author{
Mika Härkönen, ${ }^{\dagger}$ Jukka V. SePPÄLÄ, and Hannu SAlminen* $^{*}$ \\ Helsinki University of Technology, Department of Chemical Engineering, \\ FIN-02150 Espoo, Finland \\ * Borealis Polymers Oy, P.O.B. 330, FIN-06101 Porvoo, Finland
}

(Received August 22, 1994)

\begin{abstract}
A three-site model of the active sites in a $\mathrm{MgCl}_{2}$-supported Ziegler-Natta catalyst was used to simulate the effects of various external alkoxysilane donors on the catalyst active sites. The simulations correlated well with the experimental data, and it is evident that the polypropylene produced by this catalyst system originates mainly from three types of active sites. One site is a highly isospecific enantiomorphic site producing boiling heptane insoluble polymer. Two sites are responsible for the major part of the polymers of the heptane soluble firaction: a moderately isospecific enantiomorphic site (the fluctuating site) and a symmetric Bernoullian syndiospecific site. All the alkoxysilanes tested,including monoalkoxysilanes, exhibited a stabilizing role, making fluctuating sites more isospecific. In addition, the silanes having more than one alkoxy group were capable of deactivating active Ti-sites, and the selectivity of deactivation depends mainly on the size of the hydrocarbon part.
\end{abstract}

KEY WORDS Ziegler-Natta Catalysts / External Donor / Alkoxysilane / Polypropylene / Three-Site Model /

It is well known that heterogeneous ZieglerNatta catalysts contain multiple types of active sites. ${ }^{1}$ In our previous paper, ${ }^{2}$ experimental data on polymerizations of propene with various external alkoxysilane donors were correlated with two-site modelling calculations made according to a procedure introduced by Chûjô. ${ }^{3}$ Those findings supported the idea of fluctuating active sites, whose frequently changing stereospecificity produces random isotactic/ syndiotactic stereoblock copolymer. ${ }^{4-6}$ The results $^{2}$ also suggested that the polymer produced by the $\mathrm{MgCl}_{2}$-supported Ti-catalyst system actually originates from at least three types of active sites of different stereospecificity, and that the boiling heptane soluble fraction consists of polymer originating from at least two sites. The external donors were credited with two competing functions: selective deactiva- tion of active Ti-sites, and stabilization of fluctuating active sites by coordination on the $\mathrm{MgCl}_{2}$-surface nearby. The alkoxysilane donors characterized by high electron density and sterically large and protective hydrocarbon part(s), such as isobutyltrimethoxysilane, are the most effective in both selective deactivation and increasing the isospecificity of active sites.

The multi-site simulations in the recent literature $^{6-8}$ have shown that a model consisting of three sites gives a very good fit to the experimental ${ }^{13} \mathrm{C}$ NMR data. One type of site is a highly isospecific enantiomorphic site, one is a less isospecific enantiomorphic site, and one is a relatively syndiospecific site obeying Bernoullian statistics. The most isospecific site can also successfully be divided into two components leading to a four-site

\footnotetext{
† To whom correspondence should be addressed.
} 
model. $^{7}$

Although the two-site model simulations gave some evidence of fluctuating sites, ${ }^{2,4}$ the evidence was somehow speculative. As well, in our previous papers ${ }^{9-11}$ we have been inconclusive about the role of an alkoxysilane donor having only one alkoxy group: does the donor effect a highly selective deactivation or does it stabilize fluctuating sites? In this paper we carry out the simulations of three active sites as a means of finding an answer to these questions. However, this three-site model analysis is especially aimed to deepen and complete our studies on the effects of alkoxysilane donors on active sites of heterogeneous Ziegler-Natta catalysts.

\section{EXPERIMENTAL}

The catalyst was a high activity supported catalyst of type $\mathrm{MgCl}_{2} / \mathrm{TiCl}_{4} / \mathrm{DIBP} \mathrm{AlEt}_{3} /$ External donor (DIBP = diisobutyl phthalate). The polymer samples and their ${ }^{13} \mathrm{C}$ NMR data were from our earlier experiments, ${ }^{9}$ and the structures of the selected alkoxysilane donors were representative of extreme performances. The external donors were of structure $\mathrm{R}_{n} \mathrm{Si}\left(\mathrm{OR}^{\prime}\right)_{4-n}$, where $n$ is $1-3, \mathrm{R}$ is methyl, isobutyl or phenyl, and $\mathrm{R}^{\prime}$ is methyl or ethyl. The methods of the polymerizations and the NMR analysis were as described previously. ${ }^{9,10}$ The electron densities of the donors were calculated by computer assisted molecular modelling. ${ }^{12}$

The three-site model employed here is similar to that presented by Cheng. ${ }^{8}$ The parameters were fitted using the least squares technique: The difference between the calculated and observed intensity was computed for each pentad in boiling heptane soluble and insoluble fractions. The differences were then squared and their sum was calculated. The parameter values were adjusted according to the NelderMead Simplex algorithm to minimize the sum of squares. The search was terminated when the changes in both the sum of squares and the

Table I. Calculated and observed ${ }^{10}$ (in parentheses) pentad distributions of the fractions of boiling heptane extraction

\begin{tabular}{|c|c|c|c|c|c|c|c|c|c|c|}
\hline Donor $^{a}$ & $\mathrm{Si} / \mathrm{Al}^{\mathrm{b}}$ & mmmm & $m m m r$ & $r m m r$ & $m m r r$ & $m m r m+r m r r$ & rmrm & $r r r r$ & $m r r r$ & mrrm \\
\hline \multicolumn{11}{|c|}{ Boiling heptane insoluble fraction } \\
\hline No donor & 0 & $81.7(81.7)$ & $4.7(4.7)$ & $0.3(0.4)$ & $4.8(4.8)$ & $1.7(1.7)$ & $0.6(0.3)$ & $2.7(2.6)$ & $1.2(1.6)$ & $2.4(2.4)$ \\
\hline $1 \mathbf{a}$ & 0.058 & $87.9(87.9)$ & $3.0(3.2)$ & $0.2(0.3)$ & $3.2(3.1)$ & $1.2(1.3)$ & $0.4(0.3)$ & $1.5(1.5)$ & $1.0(1.0)$ & $1.6(1.4)$ \\
\hline $1 \mathbf{a}$ & 0.15 & $91.0(91.0)$ & $2.2(2.3)$ & $0.2(0.5)$ & $2.3(2.1)$ & $1.0(0.9)$ & $0.3(0.1)$ & $1.2(1.2)$ & $0.7(0.7)$ & $1.1(1.1)$ \\
\hline $\mathbf{1 a}$ & 0.2 & $91.2(91.2)$ & $2.3(2.3)$ & $0.2(0.7)$ & $2.3(2.3)$ & $0.9(0.8)$ & $0.3(0.2)$ & $1.0(1.0)$ & $0.6(0.6)$ & $1.2(1.1)$ \\
\hline $1 \mathbf{b}$ & $0.1 \varepsilon$ & $83.0(83.0)$ & $4.3(4.0)$ & $0.3(0.4)$ & $4.5(4.9)$ & $1.6(1.7)$ & $0.6(0.4)$ & $2.2(2.1)$ & $1.3(1.3)$ & $2.3(2.2)$ \\
\hline 2 & 0.1 & $92.7(92.6)$ & $1.8(1.1)$ & $0.1(0.2)$ & $1.9(2.4)$ & $0.7(0.5)$ & 0.2 & $1.1(1.0)$ & 0.6 & $1.0(1.2)$ \\
\hline 3 & $0.1 \varepsilon$ & $82.0(82.0)$ & $5.4(5.8)$ & $0.2(0.6)$ & 5.6( & & 0.5 & $1.3(1.4)$ & $1.0(0.8)$ & $2.8(1.9)$ \\
\hline 4 & $0.1 \varepsilon$ & $85.6(85.6)$ & $4.2(4.1)$ & $0.1(0.1)$ & 4.3 & 0.9 & 0.2 & $1.7(1.7)$ & $1.1)$ & $2.2(1.9)$ \\
\hline \multicolumn{11}{|c|}{ Boiling heptane soluble fraction } \\
\hline o donor & 0 & $24.3(24.0)$ & $12.9(14.8)$ & $3.1(5.0)$ & $0.1)$ & & & 10.9 & 8.4 & $6.6(7.7)$ \\
\hline 1a & 0.052 & $29.2(29.1)$ & $11.1(11.2)$ & $2.2(3.2)$ & $12.7(12.3)$ & 12. & 4.5 & 12.3 & $9.4(9.6)$ & $6.3(6.8)$ \\
\hline 1a & 0.1 & $33.3(33.3)$ & 11.1( & $1.9(2.6)$ & $12.0(11.8)$ & $10.7(1$ & 3.7 & 13.4 & $7.9(7.7)$ & $6.0(6.3)$ \\
\hline $1 \mathbf{a}$ & 0.2 & $34.4(34.4)$ & $9.8(9.7)$ & $1.6(2.3)$ & $11.1(11.5)$ & $10.4(10.1)$ & $3.2(1.6)$ & $15.2(15.1)$ & $8.6(9.4)$ & $5.6(5.9)$ \\
\hline 1b & 0.1 & $31.6(31.4)$ & $11.2(11.1)$ & $1.9(3.2)$ & $13.1(13.8)$ & $10.6(10.0)$ & $3.8(3.0)$ & $12.5(12.5)$ & $7.7(7.7)$ & $6.5(7.5)$ \\
\hline 2 & 0.1 & $32.3(32.3)$ & $11.6(12.1)$ & $1.9(2.2)$ & $12.7(12.3)$ & $10.7(11.2)$ & $3.7(2.3)$ & $12.8(12.7)$ & $8.1(8.4)$ & $6.3(6.6)$ \\
\hline 3 & 0.1 & $31.1(31.1)$ & $11.6(11.4)$ & $2.1(2.9)$ & $13.0(13.1)$ & $11.4(11.6)$ & $4.2(3.0)$ & $11.5(11.4)$ & $8.6(8.7)$ & $6.5(7.0)$ \\
\hline 4 & 0.1 & $32.4(32.4)$ & $12.1(11.6)$ & $1.9(2.7)$ & $12.8(13.3)$ & $10.5(10.7)$ & $3.8(3.0)$ & $12.6(12.6)$ & $7.4(7.3)$ & $6.4(6.6)$ \\
\hline
\end{tabular}

${ }^{a}$ The explanations of the codes are in Table II. ${ }^{b}$ Donor/AlEt ${ }_{3}$ mole ratio. 
parameters became smaller than a preset tolerance.

\section{RESULTS AND DISCUSSION}

\section{Applicability of the E/E/B Three-Site Model}

The calculated and observed pentad sequence distributions are collected in Table I. The results indicate a good fit for the $\mathrm{E} / \mathrm{E} / \mathrm{B}$ three-site model with two enantiomorphic and one Bernoullian site, even though the polymer samples were fractionated into only to two fractions. In addition, the results are in good agreement with our two-site model analysis ${ }^{2}$ which is statistically more reliable than the three-site model due to smaller number of parameters. The advantage of the three-site model is that all analysed fractions can be described with numerical values of five parameters of the model.

The active sites can be divided among one highly isospecific enantiomorphic site (E1), one less isospecific enantiomorphic site (E2) and one relatively syndiospecific site (B) obeying Bernoullian statistics. The probabilities for isospecificity of the polymer fractions produced by these sites (Table II) are in accordance with results in the recent literature. ${ }^{6,7}$

Figure 1 shows a relatively good linear correlation between the yields of isotactic polypropylene determined as boiling heptane insolubles and the productivity of the E1-sites, indicating that the boiling heptane insoluble fraction fairly well describes the most isotactic part of the polymer. Furthermore, the two-site model that we used earlier did not describe well the boiling heptane soluble fraction, ${ }^{2}$ but here the combined productivities of the E2- and B-sites exhibited a linear coorelation with the yield of boiling heptane solubles as shown in Figure 2. Together, these findings indicate that polypropylene originating from E1-sites is almost entirely insoluble in boiling heptane, while both the E2- and B-sites produce polymers soluble in boiling heptane.

This conclusion leads on to the idea that the

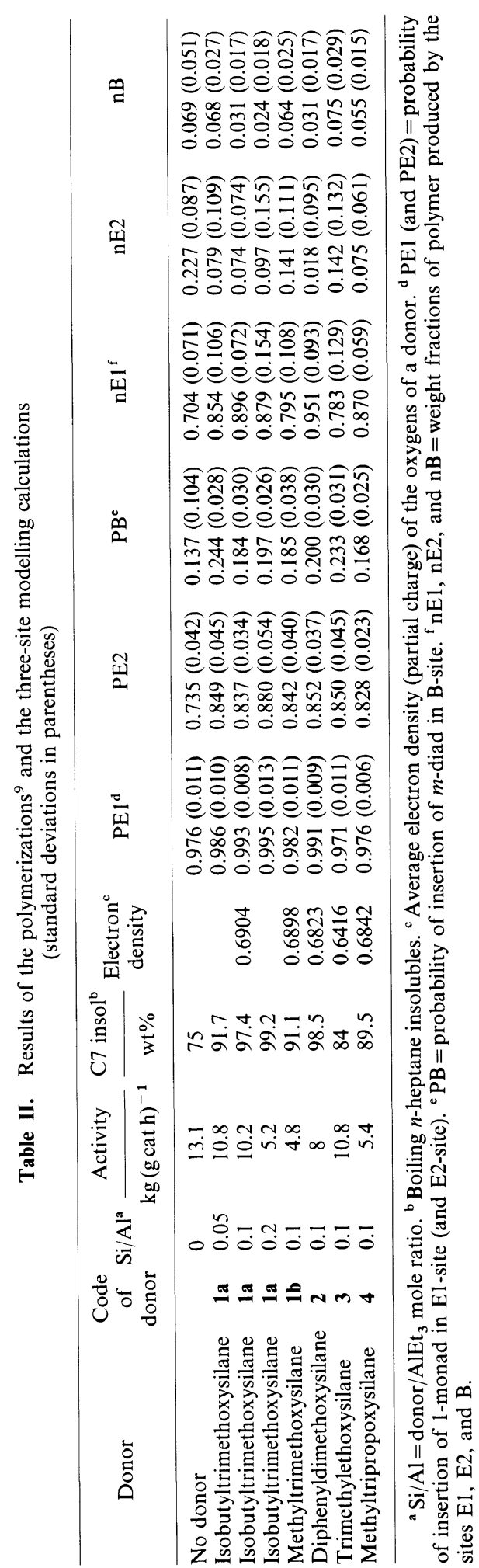

Polym. J., Vol. 27, No. 3, 1995 


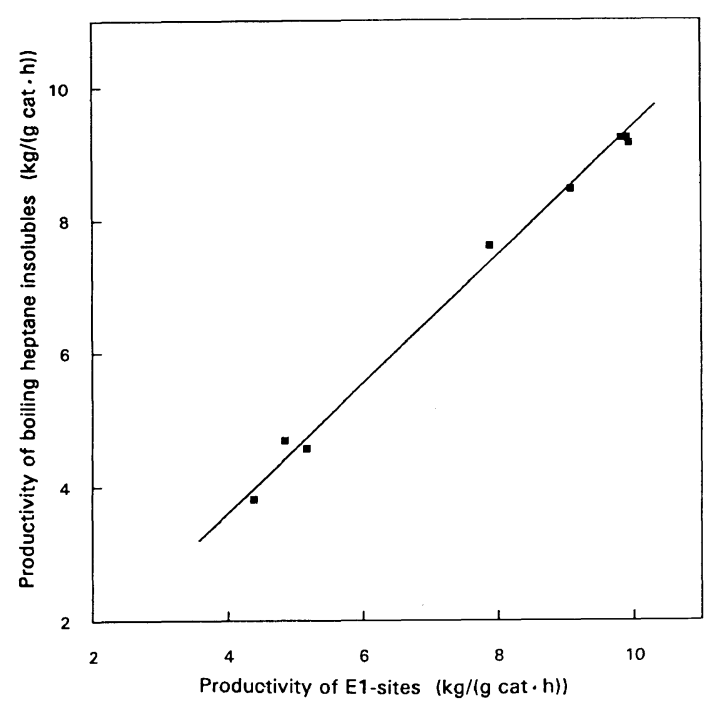

Figure 1. Comparison of the calculated productivity of E1-sites and the measured productivity of the boiling heptane insoluble fraction.

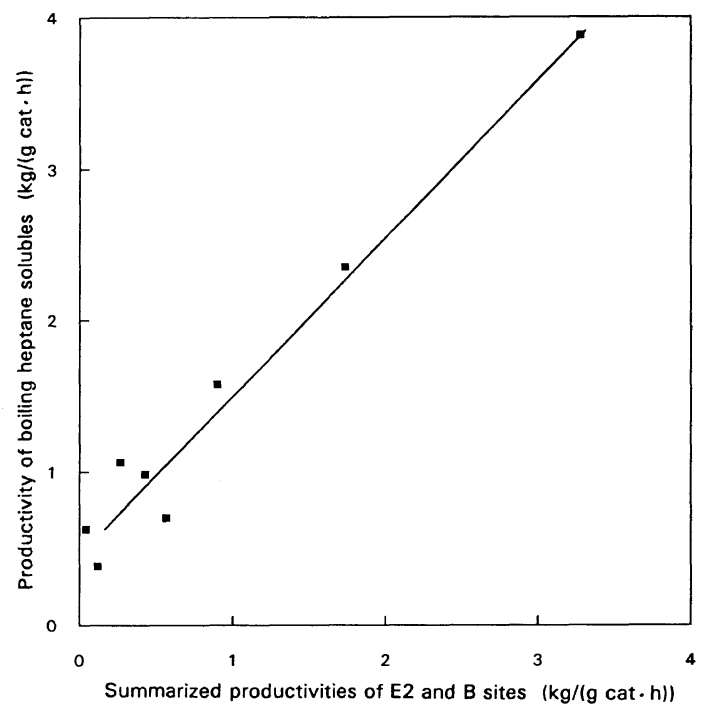

Figure 2. Comparison of the summarized calculated productivities of E1- and B-sites and the measured productivity of the boiling heptane soluble fraction.

E2-site produces relatively atactic polymer despite its enantiomorphic nature. The behaviour of E2-site match well with the proposal of fluctuating sites producing randomly chaning isotactic/syndiotactic stereoblock copolymer due to rapidly and randomly changing

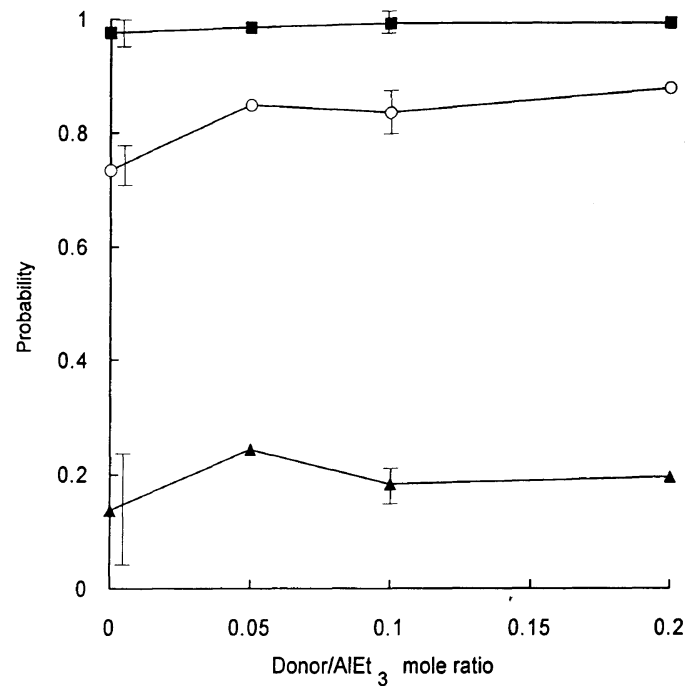

Figure 3. Effect of the silane/AlEt 3 mole ratio on the probabilities of the isospecific propagation of the E1-site $(\boldsymbol{\square})$, E2-site $(\mathrm{O})$, and B-site $(\boldsymbol{\Delta})$. Error bars describe standard deviations of the calculated probabilities.

sterical hindrance of the active Ti-compound. ${ }^{2,6,13}$

\section{Effects of External Alkoxysilane Donors on the}

\section{Active Sites}

Our results support the concept of fluctuating sites, ${ }^{4,13,14}$ The stereospecificity of the fluctuating sites changes reversibly, and it depends on whether the external donor is present or not.

As seen in Figure 3, the addition of isobutyltrimethoxysilane (1a) as an external donor increased the probability for isospecific propagation most markedly at the E2-sites. Fairly similar increase in the isospecificity of E2-sites was observed with all the tested alkoxysilane donors (1-4). The results indicate that all the external alkoxysilane donors increase the isospecificity of that site. This finding is in line with our earlier suggestions 9,10 that besides selective deactivation, which is a property strongly depending on the donor structure, all of the alkoxysilanes tested participate in formation of new kinds of isospecific active sites. The changes in stereospecificity of 
E2-sites also support a mechanism in which the increase in the isospecificity of a fluctuating site is argued to be related to the suppression of the fluctuations of the site if an external donor is located in the vicinity. $4,6,13,14$

The isospecificity of the highly isospecific E1-site was also increased upon donor addition. Although the increase was small, it was highly reproducible with good donors. The high performance donors characterized by high electron density and sterically large hydrocarbon group(s) fairly systematically gave the highest values to the probabilities of isospecific propagation of the E1-site. This finding may indicate that the E1-site actually consists of two highly isospecific sites, as proposed by Cheng. ${ }^{7}$ the increase in the isospecificity of E1 would then be explained by either stabilization or selective deactivation of the less highly isospecific site.

A slight increase was seen in the isospecificity of the B-sites as well (Figure 3). However, the changes in the probabilities were not systematic, and they fell within the limits of the standard deviations, so that the only conclusion we can draw is that the stereospecificity of the B-site is more or less unaffected by the donor. The B-site is an open symmetric site producing fairly syndiotactic polypropylene obeying Bernoullian statistics due to chain end control. External alkoxysilane donors can only deactivate these sites.

Figure 4 shows that in addition to the isospecificity enhancements deactivation of the E2- and B-sites is significant. The distribution of polymer produced by active sites $\mathrm{E} 1 / \mathrm{E} 2 / \mathrm{B}$ was $70 / 23 / 7$ with the catalyst without external donor, but it changed to $90 / 7 / 3$ when

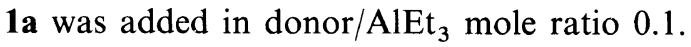
In other words the sites producing boiling heptane solubles have been considerably deactivated. The same change is seen when the productivities of the particular sites are calculated. An interesting observation in Table II is that the polymer fraction produced by E2-sites was markedly low when diphenyldi-

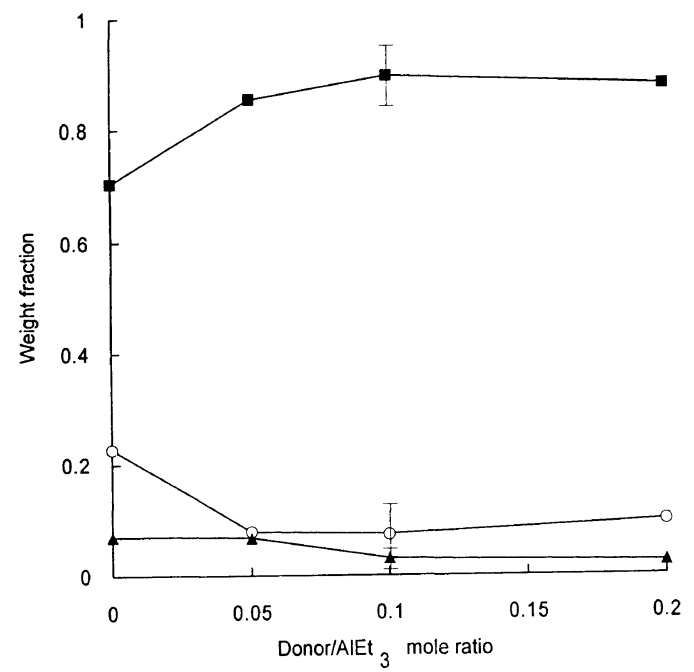

Figure 4. Effect of the silane/AlEt ${ }_{3}$ mole ratio on the weight fraction of polypropylene produced by the E1-site $(\square)$, E2-site $(\bigcirc)$, and B-site ( $\mathbf{A})$. Error bars describe standard deviations of the calculated weight fractions.

methoxysilane (2) was used as an external donor, which may indicate especially selective deactivation of E2- and B-sites. Besides selective deactivation, another possible explanation of the changes in relative amounts of active sites is that the E2-sites partially transform to E1-sites. Although such behaviour was not clearly evident in this study, the transformation mechanism cannot be totally ruled out, and perhaps this mechanism is more pronounced with dialkoxysilanes compared to trialkoxysilanes.

It has been unclear whether the monoalkoxysilanes, such as trimethylethoxysilane (3), deactivate the non-stereospecific sites or transform some sites to isospecific ones. In our most recent study we favoured the latter mechanism. ${ }^{2}$ The findings of our present work now clearly support the idea that an alkoxysilane donor having only one alkoxy group funcations primarily through stabilization of the fluctuating sites. As seen in Table II, external donor 3 increased the isotactic probability of the E2-site in the same way as the other donors, but it did not alter the 
isospecificity of either E1- or B-sites. The relative numbers of the different active sites remained almost unchanged, indicating that the single alkoxy group cannot properly deactivate active sites. A silane compound having propoxy groups (4), which has also proved to be apoor deactivator, ${ }^{9}$ affects the parameters in the same way as monoalkoxysilanes.

\section{CONCLUSIONS}

The three-site model simulations correlate very well with the experimental data, and it is evident that polypropylene produced by this $\mathrm{MgCl}_{2}$-supported Ti-catalyst system originates from at least three types of active sites of different stereospecificity. One site is a highly isospecific enantiomorphic site producing boiling heptane insoluble polymer. The other two sites, which produce the major part of the polymers of the boiling heptane soluble fraction, are a moderately isospecific enantiomorphic site (the fluctuating site) producing fairly isotactic polypropylene having heterotactic or syndiotactic stereoblocks, and a symmetric Bernoullian syndiospecific site. Some new evidence is found for the existence of the fluctuating sites and for a mechanism by which the external donor stabilizes these sites by coordinating on the catalyst surface nearby.

External donors with more than one alkoxy goup are capable of deactivating active Ti-sites, and those with a hydrocarbon part of the right size selectively deactivate the sites producing boiling heptane soluble polymers, i.e., the E2- and B-sites. In addition to selective deactivation, the alkoxysilanes with more than one alkoxy group increase the isospecificity of enantiomorphic acive sites (E1- and E2-sites).
Even external donors such as monoakoxysilanes, which are unable to effect deactivation, are effective to increase the isospecificity of the E2-sites, i.e., fluctuating sites. An external alkoxysilane donor cannot affect the stereospecificity of symmetric Bernoullian sites (B-sites).

\section{REFERENCES}

1. Y. V. Kissin, "Isospecific Polymerizaton of Olefins with Heterogeneous Ziegler-Natta Catalysis," Springer-Verlag, New York, N.Y., 1985, Chapter 4.

2. M. Härkönen, J. V. Seppälä, R. Chûjô, and Y. Kogure, Polymer (1994), in print.

3. R. Chûjô, Kagaku, 36, 422 (1981).

4. R. Chûjô, Y. Kogure, and T. Väänänen, Polymer, 35, 339 (1994).

5. V. Busico, P. Corradini, L. De Martino, F. Graziano, and A. Iadicicco, Makromol. Chem., 192,49(1991).

6. R. Paukkeri, E. Iiskola, A. Lehtinen, and $\mathrm{H}$. Salminen, Polymer, 35, 2636 (1994).

7. H. N. Cheng, Makromol. Chem., Theory Simul., 1, 415 (1992).

8. H. N. Cheng, J. Appl. Polym. Sci., 35, 1639 (1988).

9. J. V. Seppälä, M. Härkönen, and L. Luciani, Makromol. Chem., 190, 2535 (1989).

10. M. Härkönen, J. V. Seppälä, and T. Väänänen, Makromol. Chem., 192, 721 (1991).

11. M. Härkönen and J. V. Seppälä, Makromol. Chem., 192, 2857 (1991).

12. M. Härkönen, J. V. Seppälä, and L. Kuutti, Makromol. Chem., 193, 1413 (1992).

13. V. Busico, R. De Biasio, R. Cipullo, and P. Corradini, "Syndiotactic Propene Polymerization in the Presence of $\mathrm{MgCl}_{2}$-supported Ziegler-Natta Catalysts," a paper in "40 Years Ziegler Catalysts" symposium, Freiburg, Germany, 1993.

14. E. Iiskola, "Molecular Modelling Studies of Heterogeneous Stereospecific Ziegler-Natta Catalysts-The Effects of Donor Structure in Propylene Polymerization," a paper in "40 Years Ziegler Catalysts" symposium, Freiburg, Germany, 1993.

15. E. Iiskola, A. Pelkonen, H. Kakkonen, J. Pursiainen, and T. Pakkanen, Makromol. Chem., Rapid Commun, 14, 133 (1993). 